\title{
Assessing Mixing Quality of a Copovidone-TPGS Hot Melt Extrusion Process with Atomic Force Microscopy and Differential Scanning Calorimetry
}

\author{
Matthew S. Lamm, ${ }^{1,3,4}$ James DiNunzio, ${ }^{2}$ Nazia N. Khawaja, ${ }^{1}$ Louis S. Crocker, ${ }^{1}$ and Anthony Pecora ${ }^{2}$
}

Received 12 May 2015; accepted 2 August 2015; published online 18 August 2015

\begin{abstract}
Atomic force microscopy (AFM) and modulated differential scanning calorimetry (mDSC) were used to evaluate the extent of mixing of a hot melt extrusion process for producing solid dispersions of copovidone and D- $\alpha$-tocopherol polyethylene glycol 1000 succinate (TPGS 1000). In addition to composition, extrusion process parameters of screw speed and thermal quench rate were varied. The data indicated that for $10 \%$ TPGS and $300 \mathrm{rpm}$ screw speed, the mixing was insufficient to yield a singlephase amorphous material. AFM images of the extrudate cross section for air-cooled material indicate round domains 200 to $700 \mathrm{~nm}$ in diameter without any observed alignment resulting from the extrusion whereas domains in extrudate subjected to chilled rolls were elliptical in shape with uniform orientation. Thermal analysis indicated that the domains were predominantly semi-crystalline TPGS. For $10 \%$ TPGS and $600 \mathrm{rpm}$ screw speed, AFM and mDSC data were consistent with that of a single-phase amorphous material for both thermal quench rates examined. When the TPGS concentration was reduced to $5 \%$, a single-phase amorphous material was achieved for all conditions even the slowest screw speed studied (150 rpm).
\end{abstract}

KEY WORDS: atomic force microscopy (AFM); copovidone; differential scanning calorimetry (DSC); hot melt extrusion (HME); TPGS 1000.

\section{INTRODUCTION}

Hot melt extrusion, a process common to the plastic industry, represents a robust technique for preparing amorphous drug-polymer mixtures from lab-scale feasibility assessment to commercial manufacture (1-3). Because the amorphous form of the drug has greater solubility than the crystalline form, bioavailability can be increased resulting in potentially better exposure and improved patient outcomes. Typically, water-soluble polymers are used to stabilize the amorphous form of the drug in the solid state and can also prolong supersaturation during dissolution. In most cases, the goal of the extrusion process is to molecularly disperse the active pharmaceutical ingredient (API) within the polymer to yield a homogenous, single-phase, amorphous material often described as an "amorphous solid dispersion." Thus, process parameters such as screw design with its mixing, kneading and conveying elements as well as feed rates, barrel temperatures,

\footnotetext{
${ }^{1}$ Formulation Sciences Department, Merck Research Laboratories, Merck and Co., Inc., Rahway, New Jersey, USA.

${ }^{2}$ Formulation Sciences Department, Merck Research Laboratories, Merck and Co., Inc., Kenilworth, New Jersey, USA.

${ }^{3}$ Merck Research Laboratories, RY-80T-A128, 126 East Lincoln Avenue, Rahway, New Jersey 07065, USA.

${ }^{4}$ To whom correspondence should be addressed. (e-mail: matthew.lamm@merck.com)
}

screw speeds, and quench rates must all be optimized to achieve the single-phase amorphous goal. To assess if this goal has been met, the extruded material is typically analyzed with techniques that include powder X-ray diffraction, differential scanning calorimetry, FT-Raman and FT-IR spectroscopy, and solid-state NMR. Atomic force microscopy (AFM) is a scanning probe technique capable of very high spatial resolution and, when operated in tapping mode, can provide additional information such as differences in local material properties that can then be used to assess phase separation (4). The technique has been used widely in the polymer field (5) over the past two decades for evaluating systems such as polymer blends $(6,7)$, block copolymers $(8,9)$, and nanocomposites $(10,11)$. Beyond the polymer and plastic industries, the technique has been gaining popularity within the pharmaceutical field for the characterization of amorphous solid dispersions (12-16).

In this study, the utility of atomic force microscopy for evaluating the extent of mixing of a hot melt extrusion (HME) process and subsequent quenching behavior is demonstrated. Copovidone, a water-soluble, random copolymer of polyvinyl pyrrolidone and polyvinyl acetate commonly used in the pharmaceutical field to prepare amorphous solid dispersions, and $\mathrm{D}-\alpha$-tocopherol polyethylene glycol 1000 succinate (TPGS 1000), a vitamin $E$ derivative often included with the drug in these dispersions to aid in solubilization (17), were selected as model materials to assess potential phase separation. Various 
copovidone-TPGS compositions were prepared by HME while evaluating the effects of screw speed and quench rate on the extent of mixing for the process. Here, we show that AFM can assess the homogeneity of the extrudates with high spatial resolution and for cases where phase separation is detected, the size and shape of the domains. In addition, an AFM technique known as force spectroscopy was employed to measure the relative differences of mechanical properties of these domains. Finally, modulated differential scanning calorimetry (mDSC) data is provided to complement the AFM data and provide additional insight into the phase behavior of the material prepared by HME.

\section{MATERIALS AND METHODS}

\section{Materials}

Copovidone (Kollidon ${ }^{\circledR}$ VA 64) was purchased from BASF (Tarrytown, NY, USA), and TPGS 1000 was purchased from Isochem (Vert Le Petit, France). These materials were used without any further modification.

\section{Blend Preparation}

Prior to extrusion, copovidone and vitamin E TPGS were blended in a $7 \mathrm{~L}$ Bohle high shear granulator by melting vitamin E TPGS at approximately $60^{\circ} \mathrm{C}$ and slowly adding to the high shear granulator over a period of $3 \mathrm{~min}$. The blend was mixed in the granulator for an additional $90 \mathrm{~s}$ before being discharged. After discharge, the blend was milled in a Fitzmill L1A equipped with a $0.055^{\prime \prime}$ opening screen using the knives in a backward configuration at a rotational speed of $2500 \mathrm{rpm}$.

\section{Hot Melt Extrusion}

Hot melt extrusion was performed using a Leistritz ZSE $18 \mathrm{~mm}$ HP co-rotating twin screw extruder (Leistritz Corp., Somerville, NJ, USA) setup in the 40:1 L/D configuration with a $3.0 \mathrm{~mm}$ round, single bore die at the end of the unit. The screw design utilized in the study is shown in Fig. 1. Process section temperatures in zones $2-8$ were maintained at $140^{\circ} \mathrm{C}$, while zone 1 had a setpoint temperature of $105^{\circ} \mathrm{C}$. During processing, screw speeds were varied between 150 and $600 \mathrm{rpm}$. On discharge, material from the extruder was collected on a variable speed cooling conveyor or a chilled roll system (Thermoelectron, Newington, NH, USA) based on the experimental conditions shown in Table I.

\section{Atomic Force Microscopy}

Samples for AFM were prepared by scoring the extrudate with a razor blade and then fracturing to expose the cross section. The samples were then affixed to a glass slide with carbon adhesive. AFM imaging was performed with a Veeco (Santa Barbara, CA, USA) Dimension 3100 and Nanoscope V controller in tapping mode with Nanosensors (Neuchatel, Switzerland) SSS-NCHR cantilevers. The cantilevers were driven with a $5 \%$ offset from the amplitude at resonance. Feedback gains, amplitude setpoint, and drive amplitude were optimized for each sample to achieve good surface tracking. Scan rates of $1.2 \mathrm{~Hz}$ were used, and all images were collected

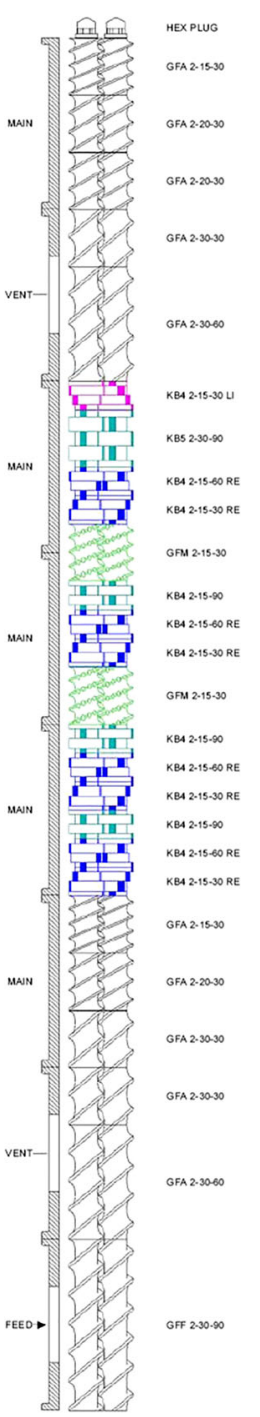

Fig. 1. Screw design for extrusion operations

with $512 \times 512$ resolution. A first-order flattening algorithm was applied to some images to compensate for sample tilt. Force spectroscopy was performed using the software's

Table I. Operating Conditions During Extrusion of Copovidone and Copovidone/TPGS Systems

\begin{tabular}{lll}
\hline TPGS level & Screw speed & Cooling method \\
\hline $0 \%$ & $150 \mathrm{rpm}$ & Air cool \\
$0 \%$ & $150 \mathrm{rpm}$ & Chilled roll \\
$0 \%$ & $600 \mathrm{rpm}$ & Air cool \\
$0 \%$ & $600 \mathrm{rpm}$ & Chilled roll \\
$5 \%$ & $150 \mathrm{rpm}$ & Air cool \\
$5 \%$ & $150 \mathrm{rpm}$ & Chilled roll \\
$5 \%$ & $300 \mathrm{rpm}$ & Air cool \\
$5 \%$ & $300 \mathrm{rpm}$ & Chilled roll \\
$10 \%$ & $300 \mathrm{rpm}$ & Air cool \\
$10 \%$ & $300 \mathrm{rpm}$ & Chilled roll \\
$10 \%$ & $600 \mathrm{rpm}$ & Air cool \\
$10 \%$ & $600 \mathrm{rpm}$ & Chilled roll \\
\hline
\end{tabular}


(Nanoscope 7.30) point and shoot feature to collect force versus displacement curves across a user-determined line drawn on the image. The photodiode detector sensitivity was calibrated by performing force measurements on a clean glass slide. For force spectroscopy, Veeco RFESP cantilevers were used with the spring constant determined using the software's thermal tune method. Force measurements were conducted with load-unload cycle of $1 \mathrm{~Hz}$ to a maximum of $700 \mathrm{nN}$.

\section{Modulated Differential Scanning Calorimetry}

A TA Instruments (New Castle, DE, USA) Q2000 differential scanning calorimeter with standard aluminum sample pans and lids was employed for the modulated DSC experiments. The sample sizes were between 5 and $11 \mathrm{mg}$. Two pinholes were placed in the lid for venting. The sample was equilibrated in the mDSC under nitrogen purge at $25^{\circ} \mathrm{C}$ for $240 \mathrm{~min}$ to drive off excess moisture and was then equilibrated at $-70^{\circ} \mathrm{C}$ before executing a heat-only modulated mDSC method with ramp rate of $2^{\circ} \mathrm{C} / \mathrm{min}$ to $175^{\circ} \mathrm{C}$ and modulation amplitude of $0.318^{\circ} \mathrm{C}$ every $60 \mathrm{~s}$. Data analysis was performed with TA Instruments Universal Analysis software. Melting endotherms were quantified using a linear baseline integration, and glass transition temperatures ( $\mathrm{Tg}$ ) were calculated as the half-height of the step transition in the reversing heat flow signal.

\section{RESULTS}

Extrusion processing was conducted under several conditions to evaluate the impact of operational variables on the ability to generate a single-phase system. During this work, material was observed for visual appearance to detect potential phase separation while also recording key in-process data to determine specific mechanical energy during processing. Operational conditions and key in-process variables recorded during production are presented in Table II. As expected, neat copovidone extruded as a clear glass rod with a slight amber color. At intermediate levels of vitamin E TPGS, the extrudate was again clear in appearance and no turbidity or hazing was observed under the screw speeds studied, suggesting a single-phase amorphous dispersion. Increasing the level of vitamin E TPGS to $10 \%$ showed extrudate with variable appearance. At lower screw speeds, independent of quenching mechanism applied, the material exhibited slight turbidity on discharge from the extruder. Increasing screw speed from 300 to $600 \mathrm{rpm}$ resulted in an appearance change of the extrudate, where material was again clear on discharge from the die.
In-process variables exhibited behaviors characteristic of the formulation properties used. In copovidone, vitamin $\mathrm{E}$ TPGS serves as a plasticizer and lowers melt viscosity in a concentration-dependent manner. As a result, motor loads decreased with higher levels of vitamin E TPGS in the formulation. Interestingly, product temperatures in the extruder exhibited values greater than the process section setpoint temperatures under high shear conditions, with plasticized formulations providing lower product temperatures under the same conditions when compared to the neat copovidone controls. This behavior was attributed to greater viscous dissipation inside the process section for the higher viscosity material and is well supported by prior reports where local temperatures may be several degrees above setpoint temperatures and mechanical energy contributes to greater than $75 \%$ of the total thermal energy within the process section. Using these values, specific mechanical energy input values were calculated (18) and are presented in Fig. 2. Similar to other in-process measurements, specific energy increased with increasing screw speed and melt viscosity.

In this study, the impact of quench rate was also studied through the use of a chilled roll system and air-cooled conveyor. During operation of the cooling conveyor, fans were left in the off position to provide the slowest cooling rate to the extrudate. Conversely, the chilled roll system was operated at a roll temperature of approximately $10^{\circ} \mathrm{C}$ to maximize cooling rate while avoiding significant roll condensation. In general, no major visual differences in material appearance were seen between the air-cooled and chilled roll product aside from the differences in extrudate shape due to processing.

The transverse cross section of extrudate from each composition/process was analyzed with atomic force microscopy operated in tapping mode simultaneously recording the height, amplitude error, and phase channels. For reference, Fig. 3 shows the cross sections of neat copovidone extruded at 600 and $150 \mathrm{rpm}$, air cooled. The topographic images (height) show a relatively flat surface with average roughness on the order of a few nanometers. The phase channel also shows a relatively uniform distribution consistent with that expected for the surface of a pure material. Images of the neat copovidone samples prepared using the chilled rolls were observed to exhibit a similar appearance (data not shown). In Fig. 4, the height and phase images for the 10\% TPGS, $600 \mathrm{rpm}$ air-cooled and chilled roll samples are presented. The images show uniform height and phase signals similar to that of the pure polymer samples, and thus, the material appears to be a single phase. No difference between the aircooled and chilled roll samples is apparent.

Table II. Compositions and Extrusion Process Parameters

\begin{tabular}{lcccc}
\hline Wt\% TPGS in copovidone & Screw speed $(\mathrm{rpm})$ & Motor load $(\%)$ & Product temp $\left({ }^{\circ} \mathrm{C}\right)$ & Melt pressure $($ Pa) \\
\hline 10 & 600 & $28.1 \pm 2.0$ & $152.2 \pm 2.7$ & $5.2 \pm 6.4$ \\
10 & 300 & $24.5 \pm 1.7$ & $140.4 \pm 2.0$ & $4.3 \pm 6.3$ \\
5 & 300 & $32.4 \pm 1.2$ & $142.9 \pm 3.0$ & $3.0 \pm 5.4$ \\
5 & 150 & $29.3 \pm 1.2$ & $139.0 \pm 0.7$ & $1.3 \pm 3.3$ \\
0 & 600 & $46.3 \pm 1.2$ & $155.1 \pm 1.0$ & $0.0 \pm 0.1$ \\
0 & 150 & $56.6 \pm 1.7$ & $150.6 \pm 0.8$ & $55.5 \pm 11.0$ \\
\hline
\end{tabular}




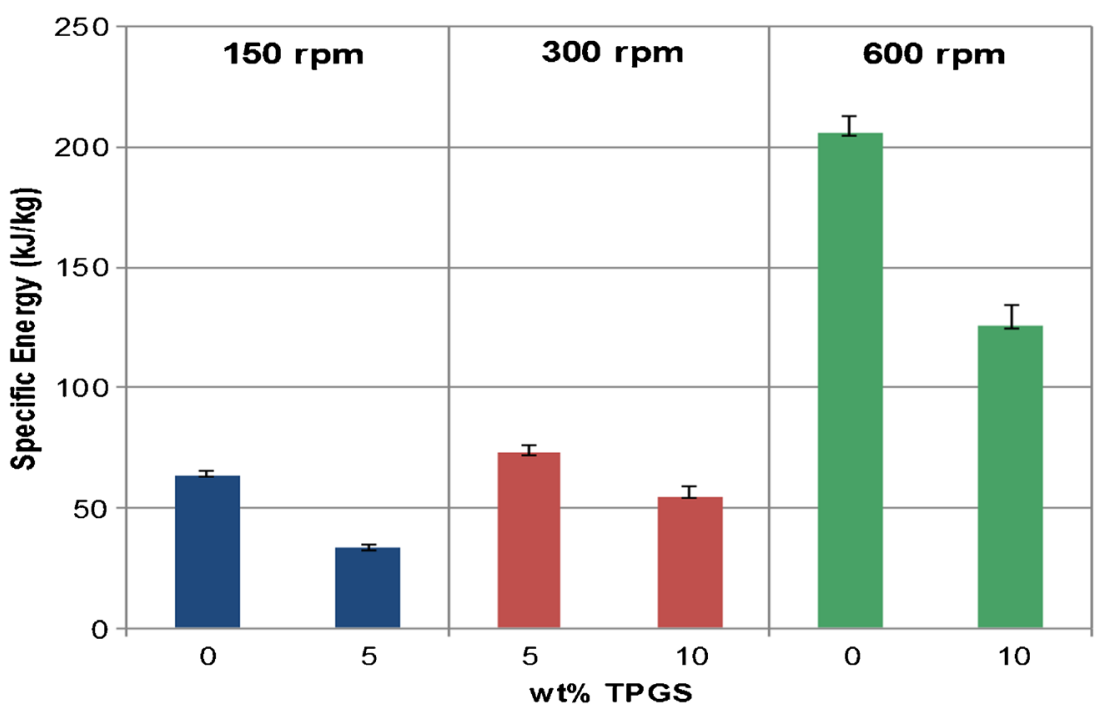

Fig. 2. Specific energy calculated for each processing condition

Figure 5 shows AFM images of the 10\% TPGS, $300 \mathrm{rpm}$ air-cooled and chilled roll samples. The height images for both cooling rates show topographic features consistent with craterlike depressions in the surface as indicated by the dark, round regions in the images ranging from approximately 200 to $800 \mathrm{~nm}$ in diameter. The depth of the depressions varies widely across samples tested from $25 \mathrm{~nm}$ to as much as a few hundred nanometers although the depth does not appear to be correlated to the diameter of a given domain. The chilled roll images in Fig. 5 show similar topographic depressions although they are elliptical in shape and oriented in the same direction as a result of localized stresses and deformation caused by thermal contraction and the rolling process. These crater-like domains also show contrast in the phase images which can be the result of differences in material properties such as modulus, adhesion, or dissipation thereby indicating the presence of two phases in the material. To reconstruct the three-dimensional shape of these domains, a longitudinal cross section (parallel to the extrusion axis) was prepared for the $10 \%$ TPGS, $300 \mathrm{rpm}$ air-cooled sample and imaged with AFM (Fig. 6). As can be seen from the height data, the domains in this projection also appear circular in shape without any observable orientation in the extrusion axis. By combining the transverse and longitudinal circular projections of the domains, it can be inferred that they must be spherical in their three-dimensional shape.
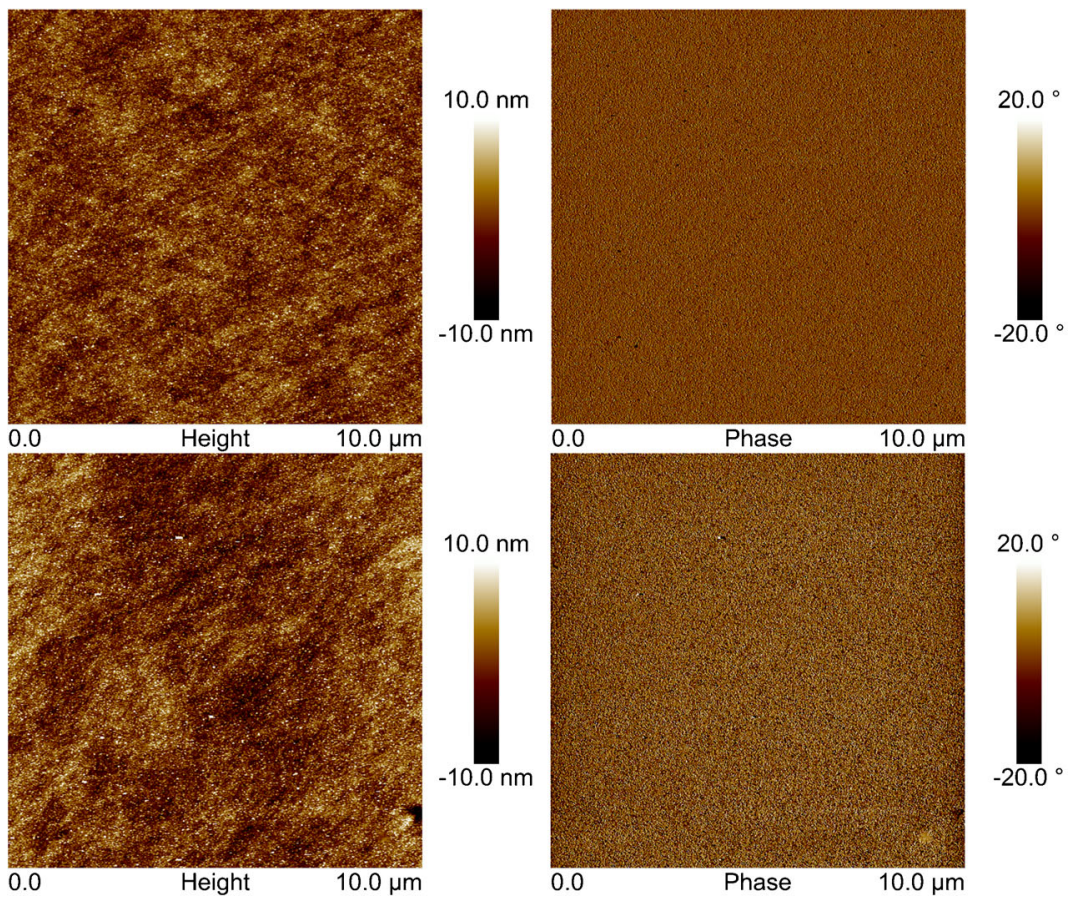

Fig. 3. AFM height (left) and phase (right) images of neat copovidone, $600 \mathrm{rpm}$ (top) and $150 \mathrm{rpm}$ (bottom); air-cooled extrudate cross sections 

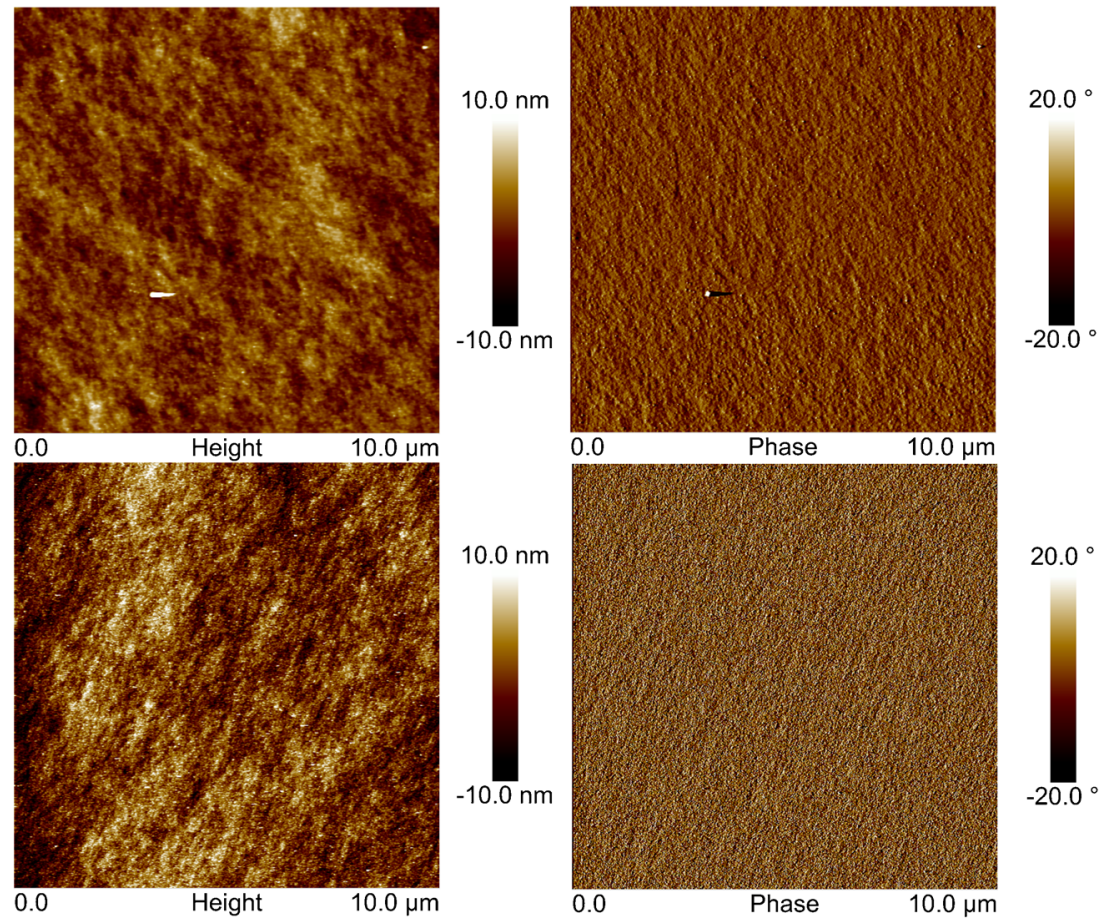

Fig. 4. AFM height (left) and phase (right) images of $10 \%$ TPGS, $600 \mathrm{rpm}$ air-cooled (top) and chilled roll (bottom) extrudate cross section

To further analyze the nature of these domains and the observed contrast in the phase signal, force-displacement curves were generated with the AFM cantilever using a technique known as force spectroscopy. In this technique, the AFM tip is brought into contact at a fixed location on the surface and then removed while measuring the cantilever deflection. With knowledge of the cantilever spring constant, in this case using the thermal tune method and calibration of the of the photodiode detector response to cantilever deflection, a force versus displacement curve can be generated. Analysis of these forcedisplacement curves can yield information about the mechanical properties of the material in this location such as stiffness and
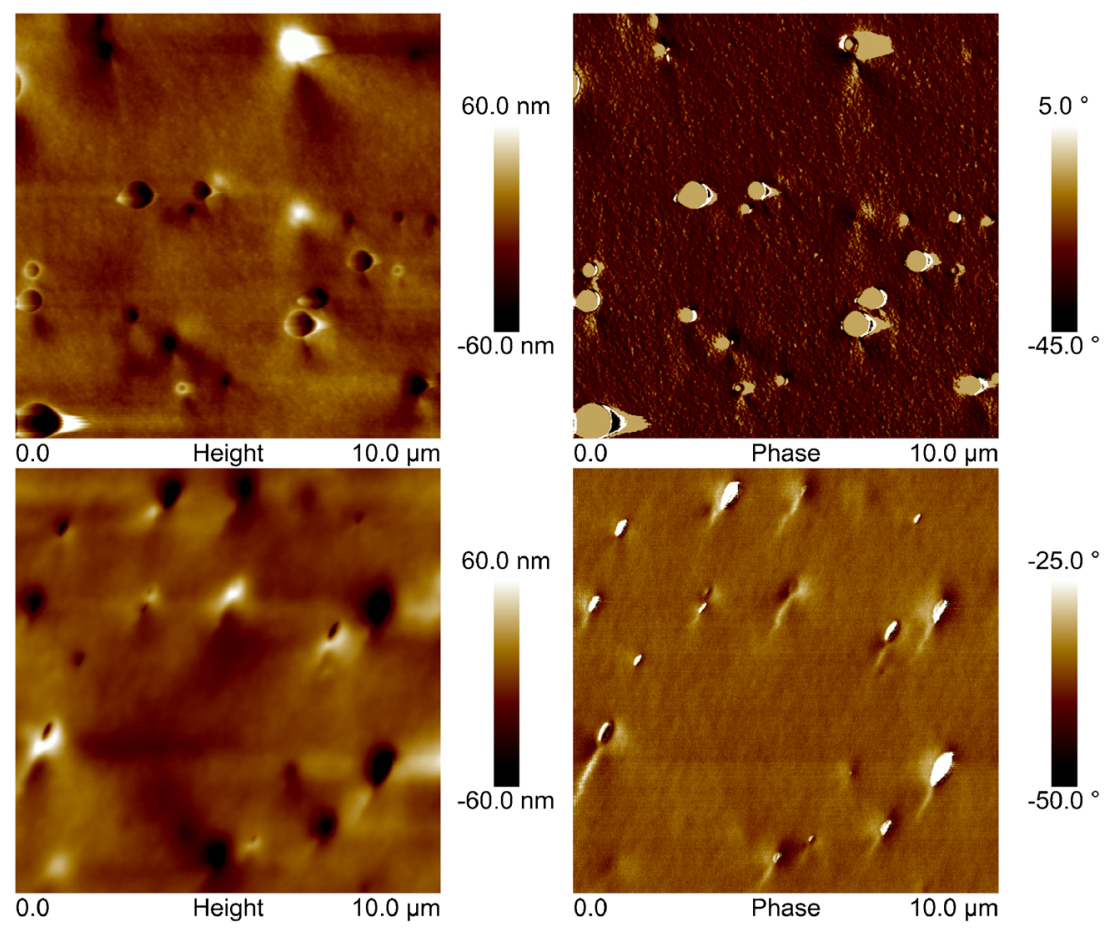

Fig. 5. AFM height (left) and phase (right) images of $10 \%$ TPGS, $300 \mathrm{rpm}$ air-cooled (top) and chilled roll (bottom) extrudate cross section 


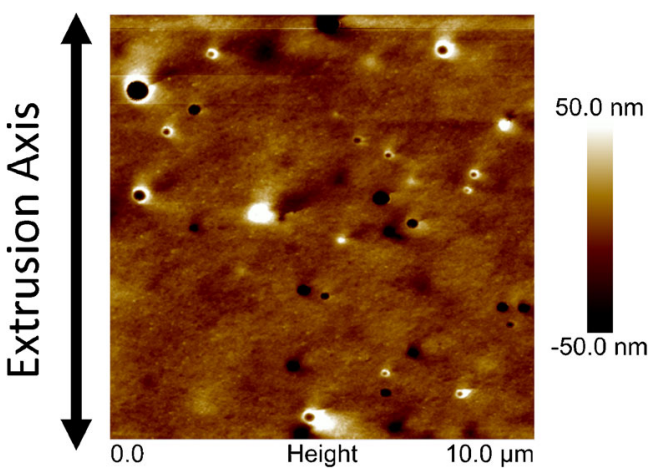

Fig. 6. AFM height image of $10 \%$ TPGS, $300 \mathrm{rpm}$ air-cooled extrudate sectioned in the longitudinal direction with respect to the extrusion axis

adhesion. Figure 7 shows an image of one of the craters from the $10 \%$ TPGS, $300 \mathrm{rpm}$, air-cooled sample in cross section. Using the AFM software's "point and shoot" feature, a line was drawn across the depression for which 25 equally spaced forcedisplacement curves were to be collected. Because of scanner and thermal drift, the actual force curves may not be in the precise location as depicted but are generally not far off. Two selected force curves: one representing the continuous phase and the other taken from inside the crater, are shown in Fig. 7. In this experiment, the tip was pressed into the surface until a maximum load of $700 \mathrm{nN}$ was reached, at which time the tip was retracted from the surface. Figure 7 shows both the approach and retract curves for measurements taken at two locations. Comparing the two force curves, it is apparent that the tip did not displace into the surface in the continuous phase as much as it did in the crater for the same maximum load. Also, the continuous phase's loading and unloading portions of the force curve are nearly identical whereas the crater phase has significant hysteresis in the curves. This indicates that the continuous phase is stiffer than the crater, i.e., less displacement under the same load, but also that the crater was plastically deformed. Whereas, the continuous phase was loaded within its elastic limits based on the hysteresis (or lack thereof) in the loadingunloading curves. Another feature of the force curves from both locations is the apparent negative force observed during the unloading curve. This is due to adhesion of the tip to the surface while trying to pull off from the surface. Positive force values indicate deflection away from the surface, while negative values represent cantilever deflection toward the surface. In addition to the adhesion seen in the unloading, there is also a slight negative force observed in the loading curve just before it increases on approach to maximum load. This negative deflection is known as "jump to contact" and is the result of attractive force pulling on the tip as the cantilever approaches the surface just before contact is made.

AFM images of the 5\% TPGS, 300, and $150 \mathrm{rpm}$, aircooled and chilled roll are shown in Figs. 8 and 9. All of the images from these samples are similar to those of the neat copovidone and therefore consistent with a single-phase material.

mDSC was performed on each sample to further understand the thermal behavior. Figure 10 shows an overlay of the reversing heat flow signals from mDSC runs on each of the samples. Both the $10 \%$ TPGS, $300 \mathrm{rpm}$ chilled roll and aircooled samples exhibit an endothermic event with onset of approximately $25^{\circ} \mathrm{C}$ and maximum of $33^{\circ} \mathrm{C}$ consistent with melting of TPGS, although pure TPGS melts with an onset of $36^{\circ} \mathrm{C}$ and maximum of $38^{\circ} \mathrm{C}$; thus, some melting point depression has occurred. The enthalpy of the $10 \%$ TPGS, $300 \mathrm{rpm}$ air-cooled endotherm was approximately $0.6 \mathrm{~J} / \mathrm{g}$, whereas the chilled roll sample was found to be $0.3 \mathrm{~J} / \mathrm{g}$. The $10 \%$ TPGS, $600 \mathrm{rpm}$ sample did not exhibit a melting endotherm nor did any of the 5\% TPGS or neat copovidone samples. For the $10 \%$ TPGS samples, a single glass transition was observed in all of the samples at approximately $80-81^{\circ} \mathrm{C}$. For the 5\% TPGS samples, a single glass transition was observed at $91-92^{\circ} \mathrm{C}$, and for the neat copovidone samples, the glass transition ranged from 107 to $110^{\circ} \mathrm{C}$.

An additional mDSC experiment was performed on a $10 \%$ TPGS, $300 \mathrm{rpm}$ air-cooled extrudate sample. Consecutive thermal cycles were performed where the temperature was cycled with an average modulated heating and cooling rate of $2^{\circ} \mathrm{C} / \mathrm{min}$ initially from -70 to 50 to $-70^{\circ} \mathrm{C}$ with each cycle increasing the maximum temperature by $25^{\circ} \mathrm{C}$. Figure 11 shows the reversing heat flow signals for the heating portions of the cycles only. The endothermic TPGS melting event in the
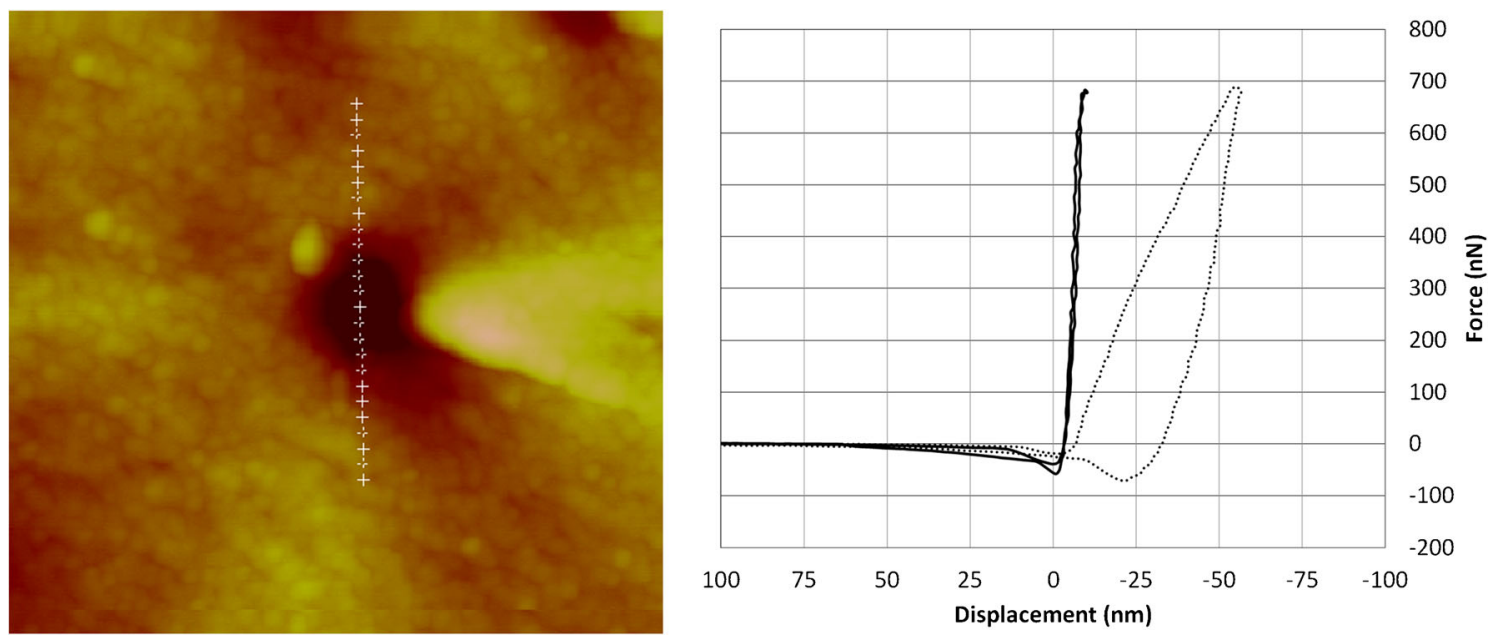

Fig. 7. Left AFM height image of $10 \%$ TPGS, $300 \mathrm{rpm}$ air-cooled sample $3 \times 3 \mu \mathrm{m}$ scan with location of force curves indicated (plus sign). Right Selected force-displacement curves from outside (solid line) and inside (dashed line) the topographic depression 

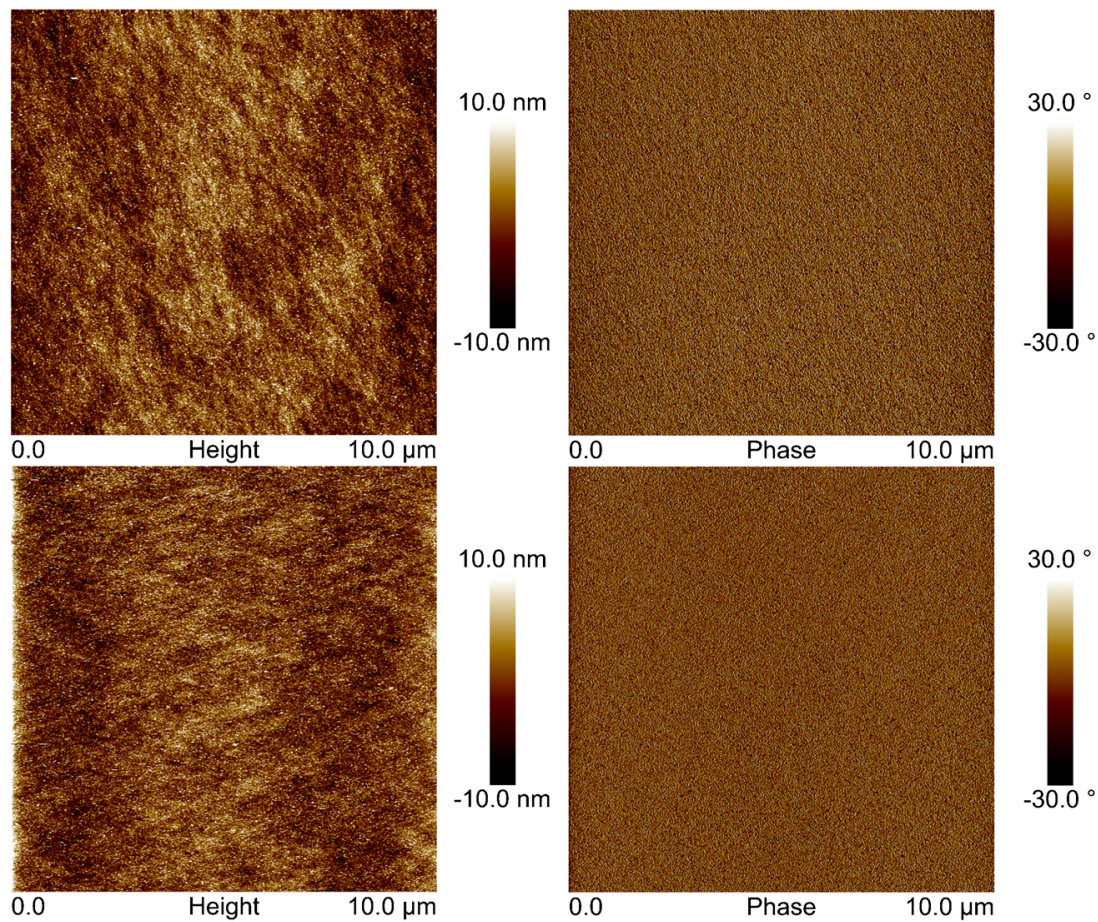

$30.0^{\circ}$

Fig. 8. AFM height (left) and phase (right) images of $5 \%$ TPGS, $300 \mathrm{rpm}$ air-cooled (top) and chilled roll (bottom) extrudate cross section

heating cycle with an onset of approximately $25^{\circ} \mathrm{C}$ persists for the first three cycles without significant change in the temperature or enthalpy of the transition. On cooling, during these cycles, there is an exothermic event associated with recrystallization of the TPGS that occurs with an onset of approximately $-23^{\circ} \mathrm{C}$ (data not shown). In the fourth heating cycle
(Fig. 11) where material had been heated to $100^{\circ} \mathrm{C}$ in the previous cycle, the endothermic melting of TPGS is observed, but the enthalpy of the transition is reduced from $0.7 \mathrm{~J} / \mathrm{g}$ in the previous cycles to $0.4 \mathrm{~J} / \mathrm{g}$. In the fifth heating cycle, a small endotherm is observed with only $0.1 \mathrm{~J} / \mathrm{g}$ enthalpy, and in the sixth cycle, there is no endotherm detected. Thus, as the
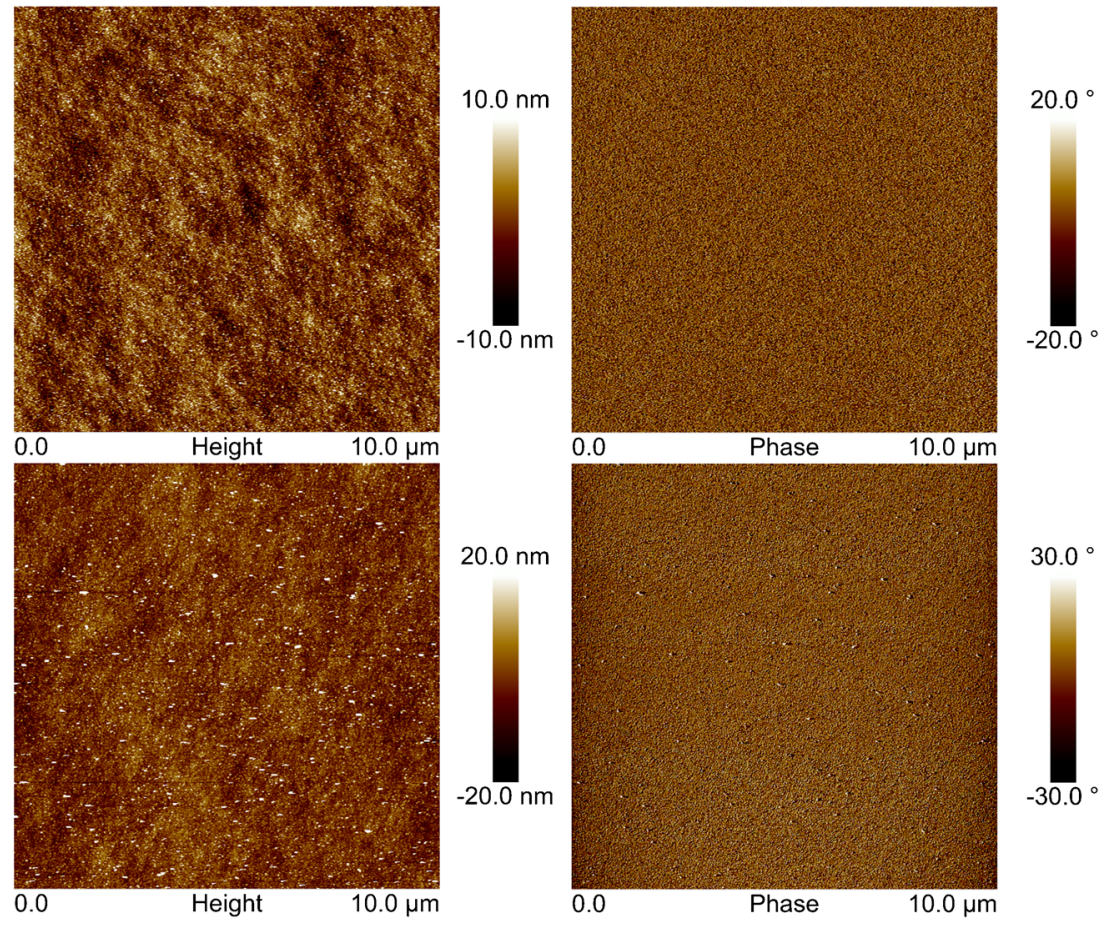

Fig. 9. AFM height (left) and phase (right) images of 5\% TPGS, $150 \mathrm{rpm}$ air-cooled (top) and chilled roll (bottom) extrudate cross section 


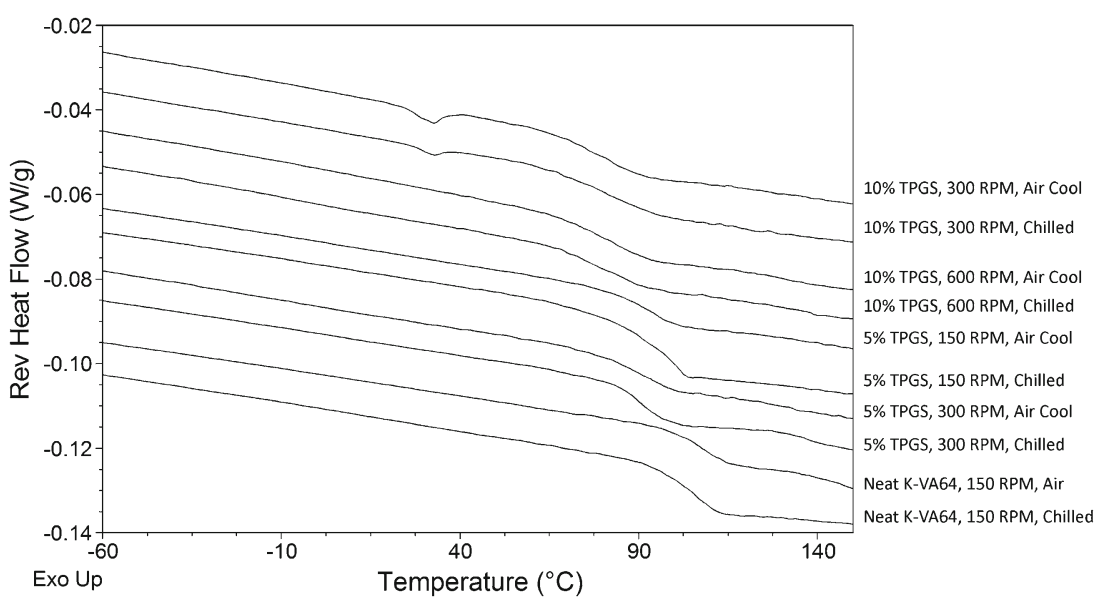

Fig. 10. Overlay of reversing heat flow signals from modulated differential scanning calorimetry of each sample

material is heated above $100^{\circ} \mathrm{C}$, the amount of material that recrystallizes becomes reduced until heating to $150^{\circ} \mathrm{C}$ results in no recrystallization during cooling or melting on reheating. Despite the changes in melting enthalpy, the $\mathrm{Tg}$ of $79^{\circ} \mathrm{C}$ for fourth, fifth, and sixth heating cycles remained unchanged.

\section{DISCUSSION}

The data presented herein clearly show the effect of hot melt extrusion processing conditions on the resulting phase behavior of the extrudate and the ability of atomic force microscopy to detect phase separation within the materials. Since a single phase was observed for all the 5\% TPGS in copovidone samples, regardless of processing conditions tested, the focus of this discussion will be on the 10\% TPGS in copovidone samples where phase separation was observed for the $300 \mathrm{rpm}$ screw speed and a single phase for the $600 \mathrm{rpm}$ screw speed. Thus, the screw speed for this system appears to be a significant factor in determining the extent of mixing and the ability to achieve a single phase. Based on the AFM data, it is apparent that with the $300 \mathrm{rpm}$ screw speed, the material was not completely mixed as it exited the extruder as opposed

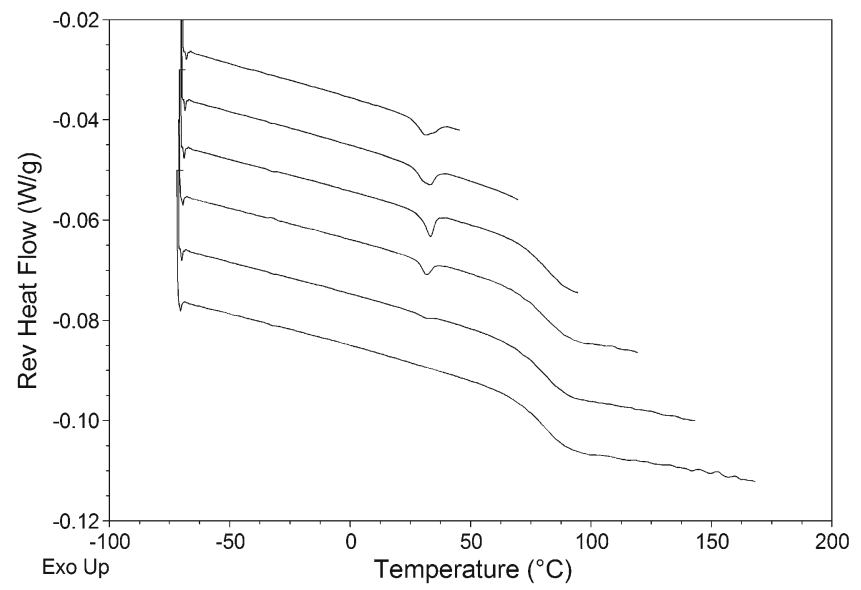

Fig. 11. Thermal cycling of the $10 \%$ TPGS, 300 rpm air-cooled sample. Reversing heat flow signals are presented for the heating cycles only (offset for clarity) to phase separating post extrusion. This is supported by the chilled roll AFM images where the domains were elliptical in shape due to the flattening of the extrudate by the rolls; that is to say that the domains had to exist before entering the rolls in order to be misshapen. The effect of increasing screw speed for a given material feed rate on the hot melt extrusion process has multiple effects. First, the mechanical energy input to the material is increased with screw speed. More importantly, the distributive and dispersive mixing of the material is increased with screw speed under a fixed feed rate. The residence time distribution of material in the extruder will remain largely unchanged due to operation at a fixed feed rate; however, shifts in the shape of the distribution are expected. While these differences may shift cumulative distributions, the overall impact is not expected to exceed a timescale of seconds whereas thermal measurements by mDSC can occur over many minutes. Interestingly, the thermal cycling mDSC data in Fig. 11 indicates that diffusive mixing can occur without mechanical mixing as the temperature is increased above $125^{\circ} \mathrm{C}$. However, the heating rate of $2^{\circ} \mathrm{C} / \mathrm{min}$ used in the mDSC experiment leads to material exposed to elevated temperatures for much longer periods of time as compared to the time that the material was at elevated temperature $\left(\sim 140^{\circ} \mathrm{C}\right)$ in the extruder. Thus, given the same feed rate, barrel temperature, and screw speed of $300 \mathrm{rpm}$, but a longer barrel length, i.e., longer residence time distribution comparable to the mDSC experiment, complete mixing would be expected to occur.

Preparations of solid dispersions containing binary mixtures of copovidone and TPGS 1000 have been reported previously. Yoo et al. prepared a 10\% TPGS in a VA64 dispersion by rotary evaporation and reported crystallinity observed by polarized light microscopy and DSC (19). Van den Mooter et al. used spray draying to prepare dispersions containing 10, 25, and 40\% TPGS 1000 in copovidone (20). They found that the $10 \%$ TPGS 1000 sample was amorphous by PXRD and contained a single $\mathrm{Tg}$ at $85^{\circ} \mathrm{C}$ as determined by modulated DSC. Increasing the loading to $25 \%$ yielded phaseseparated material with a melting endotherm at $32.6{ }^{\circ} \mathrm{C}$ and two Tgs observed in the mDSC. Thus, it appears that they also observed melting point depression of the TPGS in this study though it was not discussed specifically. In another study 
involving TPGS and Eudragit E100, they again observed TPGS melting point depression and attributed it to the Eudragit E100 inducing chain folding of the polyethylene glycol side chains in TPGS (21). Although this is a plausible explanation, in our review of the literature, we have not found specific evidence via X-ray, neutron diffraction, or any other method demonstrating that the relatively short TPGS 1000 PEG chains have crystallized in a folded conformation. In studies of low molecular weight polyethylene oxide, chainfolded crystals were found to be unstable below $3000 \mathrm{MW}$ (22). Other possible explanations of the observed melting point depression could be due simply to the miscibility of TPGS in copovidone, i.e., a negative Flory-Huggins $x$ parameter or that the presence of the polymer in the TPGS domains yields crystallites of small size and/or imperfect quality that melt at a lower temperature (23). In any case, it is clear from these reports and our data that the phase behavior of a $10 \%$ TPGS 1000 in copovidone solid dispersion is dependent on preparation method and processing parameters.

Noting the similar thermal histories experienced by the material at 300 and $600 \mathrm{rpm}$ and the preceding discussion about process modality impact on phase behavior, it is clear that polymer-polymer diffusion cannot solely explain the resulting phase behavior of the dispersion. Under processing conditions where the process section temperature is above the melting temperature of TPGS 1000 and glass transition temperature of copovidone, the process is one of mixing a low viscosity additive (TPGS 1000) with a high viscosity phase (copovidone). In this case, TPGS 1000 is suspended within the copovidone phase where the low-viscosity droplets take on a characteristic size due in part to the initial distribution of TPGS in the feed blend. As droplets transition through the process section and across the kneading/mixing regions, the droplets are deformed and broken as a result of strain to a final striation thickness that is inversely proportional to total strain within the system (24). At higher screw speeds, where greater specific mechanical energies were observed, the increased total strain results in smaller droplets of vitamin $\mathrm{E}$ TPGS at $600 \mathrm{rpm}$ that can successfully diffuse into the polymer over the timescale of the extrusion operation, while at lower screw speeds, the larger droplets are unable to fully diffuse into the external phase on the timescale of the extrusion process.

Another interesting aspect of this study was the investigation of quenching mechanism on phase behavior. Although both quenching modalities resulted in a $10 \%$ vitamin E TPGS formulation that showed phase separation when processed at $300 \mathrm{rpm}$, the mechanism of quenching influenced domain shape and also apparent size. As discussed previously, the presence of the droplets in both systems suggests that the material was present on discharge from the die. This is corroborated with the visual observations of turbidity under the extrusion conditions. More interesting is the difference in melting enthalpy of the residual vitamin E TPGS domain, which was greater for the air-cooled sample. It has been previously suggested that HME can be described by two processes: a mixing at elevated temperature and a quenching process to lock the dispersion properties by rapidly transitioning to below the glass transition temperature. During the period of the quenching process where the system is above the $\mathrm{Tg}$ and below the component solubilization temperature, it is possible that recrystallization could occur. In such a case where residual vitamin E TPGS serves as a nucleation point for continued phase separation, a reduced quench rate may result in greater levels of the separated phase. Although additional work is necessary to confirm this behavior, it does point to a potential impact of quenching rate on dispersion uniformity from melt extruded systems.

The use of atomic force microscopy for characterizing pharmaceutical solid dispersions prepared by hot melt extrusion has been previously reported. For example, Lauer et al. have used AFM to characterize the homogeneity of drug-polymer mixtures prepared using a microhot melt extruder (16) and compared the data to dispersions prepared using a screening method involving solvent casting. Qi and Craig have utilized AFM coupled with local thermal analysis as well as photothermal Fourier-transform infrared (PT-FTIR) microspectroscopy to characterize their drug-loaded hot melt extrudates (25). These studies, among others, and our report herein demonstrate the power of AFM and related techniques to characterize the effect of processing and composition on the resulting phase behavior of hot melt extrudate for pharmaceutical applications.

\section{CONCLUSIONS}

In this study, the utility of atomic force microscopy to characterize the phase behavior and morphology of solid dispersions comprised of copovidone, and TPGS 1000 prepared by hot melt extrusion was demonstrated. The effects of processing parameters including screw speed and cooling method were studied as well as the compositional effects on the resulting hot melt extrudate. At $10 \%$ by weight loading of TPGS 1000 in copovidone, a single phase was observed by AFM and mDSC for the screw speed of $600 \mathrm{rpm}$. However, a screw speed of $300 \mathrm{rpm}$ for the same composition yielded material that was observed to be phase separated by AFM and mDSC. Thus, the slower screw speed resulted in insufficient mixing and yielded a product that was phase separated on exiting the extruder. Rapid cooling via the chilled roll resulted in reduced crystallinity of the phase-separated TPGS as compared to the slower cooled sample. Reducing the TPGS 1000 loading to $5 \%$ by weight resulted in singlephase material generated with both screw speeds (300 and $150 \mathrm{rpm}$ ) and cooling rates.

\section{REFERENCES}

1. Almeida A, Claeys B, Remon JP, Vervaet C. Hot melt extrusion developments in the pharmaceutical industry. In: Douroumis D, editor. Hot-melt extrusion: pharmaceutical applications. Chichester, UK: John Wiley \& Sons, Ltd; 2012; p. 43-69.

2. Crowley MM, Zhang F, Repka MA, Thumma S, Upadhye SB, Kumar Battu S, et al. Pharmaceutical applications of hot-melt extrusion: part I. Drug Dev Ind Pharm. 2007;33(9):909-26. doi:10.1080/03639040701498759.

3. Repka MA, Langley N, DiNunzio J. Melt extrusion: materials, technology and drug product design. New York: Springer; 2013.

4. Yablon DG, Haugstad G. Phase imaging. In: Yablon DG, editor. Scanning probe microscopy in industrial applications: nanomechanical characterization. Hoboken, New Jersey: John Wiley \& Sons, Inc.; 2013. p. 95-114.

5. Tsou AH, Yablon DG. Atomic force microscopy of polymers. In: Yablon DG, editor. Scanning probe microscopy in industrial applications. Hoboken, New Jersey: John Wiley \& Sons, Inc.; 2014. p. 210-31. 
6. Bar G, Thomann Y, Brandsch R, Cantow HJ, Whangbo MH Factors affecting the height and phase images in tapping mode atomic force microscopy. Study of phase-separated polymer blends of poly(ethene-co-styrene) and poly(2,6-dimethyl-1,4phenylene oxide). Langmuir. 1997;13(14):3807-12.

7. Boltau M, Walheim S, Mlynek J, Krausch G, Steiner U. Surfaceinduced structure formation of polymer blends on patterned substrates. Nature. 1998;391(6670):877-9.

8. McLean RS, Sauer BB. Tapping-mode AFM studies using phase detection for resolution of nanophases in segmented polyurethanes and other block copolymers. Macromolecules. 1997;30(26):8314-7.

9. Kim SH, Misner MJ, Xu T, Kimura M, Russell TP. Highly oriented and ordered arrays from block copolymers via solvent evaporation. Adv Mater. 2004;16(3):226-31.

10. Zhang MQ, Rong MZ, Zeng HM, Schmitt S, Wetzel B, Friedrich K. Atomic force microscopy study on structure and properties of irradiation grafted silica particles in polypropylene-based nanocomposites. J Appl Polym Sci. 2001;80(12):2218-27.

11. Chow W, Ishak Z, Karger-Kocsis J. Atomic force microscopy study on blend morphology and clay dispersion in polyamide-6/ polypropylene/organoclay systems. J Polym Sci B Polym Phys. 2005;43(10):1198-204.

12. Lamm MS. Applications of scanning probe microscopy and nanomechanical analysis in pharmaceutical development. In: Yablon DG, editor. Scanning probe microscopy in industrial applications. Hoboken, New Jersey: John Wiley \& Sons, Inc.; 2014. p. 287-301.

13. Lauer M, Grassmann O, Siam M, Tardio J, Jacob L, Page S, et al. Atomic force microscopy-based screening of drug-excipient miscibility and stability of solid dispersions. Pharm Res. 2011;28(3):572-84. doi:10.1007/s11095-010-0306-4.

14. Six K, Murphy J, Weuts I, Craig DM, Verreck G, Peeters J, et al. Identification of phase separation in solid dispersions of itraconazole and Eudragit $\hat{A} \circledast$ E100 using microthermal analysis. Pharm Res. 2003;20(1):135-8.

15. Van Eerdenbrugh B, Lo M, Kjoller K, Marcott C, Taylor LS. Nanoscale mid-infrared imaging of phase separation in a drugpolymer blend. J Pharm Sci. 2012;101(6):2066-73.
16. Lauer M, Siam M, Tardio J, Page S, Kindt J, Grassmann O. Rapid assessment of homogeneity and stability of amorphous solid dispersions by atomic force microscopy-from bench to batch. Pharm Res. 2013;30(8):2010-22. doi:10.1007/s11095-013-1045-0.

17. Guo YY, Luo J, Tan SW, Otieno BO, Zhang ZP. The applications of Vitamin E TPGS in drug delivery. Eur J Pharm Sci. 2013;49(2):175-86. doi:10.1016/j.ejps.2013.02.006.

18. Steiner R. Extruder design. In: Ghebre-Selassie I, Martin C, editors. Pharmaceutical extrusion technology. New York: Marcel Dekker, Inc.; 2003. p. 17-35.

19. Yoo S, Krill SL, Wang Z, Telang C. Miscibility/stability considerations in binary solid dispersion systems composed of functional excipients towards the design of multi-component amorphous systems. J Pharm Sci. 2009;98(12):4711-23.

20. Janssens S, Nagels S, Armas HN, D’Autry W, Van Schepdael A, Van den Mooter G. Formulation and characterization of ternary solid dispersions made up of Itraconazole and two excipients, TPGS 1000 and PVPVA 64, that were selected based on a supersaturation screening study. Eur J Pharm Biopharm. 2008;69(1):158-66.

21. Goddeeris C, Willems T, Houthoofd K, Martens JA, Van den Mooter G. Dissolution enhancement of the anti-HIV drug UC 781 by formulation in a ternary solid dispersion with TPGS 1000 and Eudragit E100. Eur J Pharm Biopharm. 2008;70(3):861-8. doi:10.1016/j.ejpb.2008.07.006.

22. Buckley C, Kovacs A. Melting behaviour of low molecular weight poly (ethylene-oxide) fractions. Colloid Polym Sci. 1976;254(8):695715.

23. Rim PB, Runt JP. Melting point depression in crystalline/ compatible polymer blends. Macromolecules. 1984;17(8):1520 6.

24. Tadmor Z, Gogos CG. Mixing. Principles of polymer processing. Hoboken, New Jersey: John Wiley \& Sons, Inc.; 2013. p. 322-91.

25. Qi S, Belton P, Nollenberger K, Gryczke A, Craig DM. Compositional analysis of low quantities of phase separation in hot-melt-extruded solid dispersions: a combined atomic force microscopy, photothermal fourier-transform infrared microspectroscopy, and localised thermal analysis approach. Pharm Res. 2011;28(9):2311-26. doi:10.1007/s11095-011-0461-2. 